\author{
Military Technical College \\ Kobry El-Kobbah, \\ Cairo, Egypt.
}

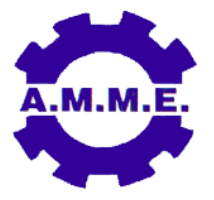

\author{
$16^{\text {th }}$ International Conference \\ on Applied Mechanics and \\ Mechanical Engineering.
}

\title{
AEROELASTIC OPTIMIZATION OF FUNCTIONALLY GRADED WINGS
}

\author{
A. M. El-Gohary ${ }^{*}$, K. Y. Maalawi ${ }^{* *}$ and H. M. Negm ${ }^{* \star *}$
}

\begin{abstract}
This paper presents a model for aeroelastic optimization of functionally graded, plate subsonic wings. The objective is the maximization of the critical flight speed at which wing divergence occurs, while maintaining the total structural mass at a constant value equals to that of a known baseline design. The major aim of the study is to tailor the fiber volume fraction distribution in order to improve the wing aeroelastic performance and broaden its stability boundaries without mass penalty. Various power-law mathematical expressions describing material grading along the wing span as well as the airfoil thickness directions have been utilized, where the power exponent is taken as a main design variable. The pre-assigned aerodynamic parameters are chosen to be the wing area, aspect ratio and chord taper ratio. The mathematical model employs the classical plate and beam theories for determining elastic deformations of the wing structure, and the modified strip theory for calculating the aerodynamic loads that arise from these deformations. This representation, together with the classical lamination theory, allows the solution of the wing divergence problem using the finite element method. The resulting optimization problem has been solved by invoking the MATLAB optimization Toolbox routines, which implement the sequential quadratic programming method. Adequate scaling and non-dimensionalization of the various parameters and variables are utilized in order to make the model valid for a variety of wing configurations and types of material of construction. A case study involving the optimization of a tapered plate subsonic wing made of carbon-AS4/epoxy-3501-6 composites is presented. Trends for good designs having expanded aeroelastic stability boundary under the imposed mass constraint are discussed. Results show that the approach implemented in this study can be efficient in producing improved designs in a reasonable computer time.
\end{abstract}

\section{Keywords}

Structural optimization, Material grading, Aeroelasticity, Divergence, Finite elements.

* Egyptian Armed Forces. Email: ahmed elgohary262@yahoo.com.

*** Mech. Eng. Dept., National Research Center, Maalawi@hotmail.com.

Professor of Aircraft Structures, Cairo University, Giza, Egypt. 


\section{NOMENCLATURE}

$\begin{array}{ll}\mathrm{C} & \begin{array}{l}\text { Chord length } \\ \mathrm{C}_{0}, \mathrm{H}_{0}\end{array} \\ \hat{C}_{j}=\left(C_{j} / C_{0}\right) & \text { Chord and depth of wing baseline cross section } \\ \widehat{H}_{j}=\left(H_{j} / H_{0}\right) & \text { Normalized mean chord of the } j \text {-th finite element } \\ \hat{h}_{i j}=\left(h_{i j} / H_{0}\right) & \begin{array}{l}\text { Normalized thickness of the } i \text {-th layer in the } j \text {-th element, } \\ i=1,2, \ldots, N_{L, j}\end{array} \\ \hat{L}_{j}=\left(L_{j} / L\right) & \text { Normalized length of the } j \text {-th finite element } \\ \mathrm{M}_{0} & \text { Total mass of baseline design } \\ N_{E} & \text { Number of finite elements } \\ N_{L, j} & \begin{array}{l}\text { Average value of the number of layers in the } j \text {-th finite element, } \\ j=1,2, . N_{E}\end{array} \\ V_{f, i j} & \text { Fiber volume fraction in the } i \text {-th layer in the } j \text {-th element } \\ \theta_{i} & \text { Fiber orientation angle in the } i \text {-th layer }\end{array}$

\section{INTRODUCTION}

With the growing demand for cost-effective designs of several types of aerospace, civil and mechanical structures, optimization of structural components has been gaining increasing attention for its acknowledged design enhancement, especially in the early stages of product development. One of the most important design issues in aerospace industry is the aeroelastic instabilities that may arise from the interactions among aerodynamic, inertial and structural loads applied to a flight vehicle. The interaction of at least two of these loads produces various aeroelastic phenomena, such as divergence, flutter, control reversal, buffeting and dynamic instability [1]. These instabilities result in reduced control of the flight vehicle, undesirable performance, and often cause damage, sometimes catastrophic, to the vehicle structure. Therefore, by incorporating aeroelastic considerations into an early design optimization, the design space of the flight vehicle will be reduced such that undesirable aeroelastic effects can be avoided during the range of the vehicle's mission profile. Actually, design optimization of an airframe involves many objectives, constraints and design variables. This is because the structure of a flight vehicle contains thousands of components ranging from small rivets and bolts to large, heavyweight bulkheads and spars.

In general, the application of design optimization to a complicated aero-structure system, like an aircraft wing, takes place in two stages. The first is called the lowfidelity stage, and the second is the high-fidelity stage [2]. Several pioneer applications in the context of aeroelastic optimization of aircraft structures can be 
found in the literature. Butler et al. [3] calculated the minimal mass design of a high aspect ratio composite wing under the condition that both divergence and flutter speeds exceed certain low limiting values. The wing was modeled as a series of box beams, whilst aeroelastic loads were based on the aerodynamic strip theory. Design variables encompassed engine position, spars locations as well as laminate ply thickness variation. Peter J. et al. [4] presented a combined aerodynamic and structural optimization model of a high-speed civil transport wing. Design objectives included minimum aerodynamic drag and structural weight subjected to constraints imposed on torsional divergence, strength, and buckling. Another work by Layton [5] applied multi-objective optimization to find the optimal static and dynamic aeroelastic design of a swept-back wing with the design variables selected to be the sweep-back angle, aspect ratio and chord distribution.

Another new class of composite materials can be produced by varying the volume fractions of their constituents in a predetermined profile. Such non-uniform composites are called functionally graded materials (FGMs), in which the properties are functions of the spatial coordinates [6]. FGMs may be defined as advanced composite materials fabricated to have graded variation of the volume fractions of the constituent materials. They were originated in Japan in 1984 during the space project, in the form of proposed thermal barrier material capable of withstanding high temperature gradients. Cho and Oden [7] optimized the transient and steady-state thermal stresses in a ceramic-metal FGM, where the gradation was represented by a two-parameter curve with the coefficients serving as design variables. Another work by Cho and Shin [8] employed a back propagation artificial neural network to achieve an optimum material composition in a three-layered plate consisting of ceramic and metal layers and a FGM layer sandwiched between them. Limited work may be found dealing with FGMs applications in the context of aeroelasticity. Librescu and Maalawi $[9,10]$ investigated optimization of composite wings using the concept of grading in either material or shear wall thickness. The objective was maximization of the divergence speed, while maintaining the total structural weight at a value equal to that of a baseline design. Both continuous and discrete distributions of the volume fractions of the constituent materials were analyzed using analytical Bessel functions of the first and second kind. It was shown that global optimality solutions can be achieved for a variety of wing configurations. The method of Sequential Quadratic Programming (SQP) [11], coupled with the developed finite element code is used to obtain the needed optimal wing designs. The main motivation for this research work was to utilize the concept of material grading for enhancing the aeroelastic performance of a straight, tapered, high aspect ratio composite wing. The aim is to tailor the structural mass and stiffness distributions in such a way as to raise the stability boundary of the wing without weight penalty at an early design stage.

In the present work, a simplified model is formulated by maximizing the critical flight speed at which wing divergence occurs. The total structural mass is maintained at a fixed value equal to that of a baseline design in order not to violate other performance requirements of the aircraft. While there are other important aeroelastic phenomena, divergence speed is used here as an acceptable measure of the overall torsional stiffness level of the wing structure. This can be a key factor in designing forward swept wings which are prone to static aeroelastic instability rather than dynamic instabilities such as flutter or buffeting. The selected design variables include the volume fractions of the constituent materials of construction as well as 
geometric and cross-sectional parameters of the wing. Considering manufacturing restrictions, side constraints are also set on the fiber volume fraction in order not to violate prescribed lower and upper limiting values.

\section{FINITE ELEMENT FORMULATION}

Figure (1) shows the normalized wing configuration where all variables are nondimensionalized with respect to those of a known baseline design. The platebeam model is assumed to be divided into a preassigned number of panels covering the inboard, middle and outboard regions of the wing surface. Each panel is subdivided into a reasonable number of finite elements as shown in the figure. The various wing parameters are defined as follows:

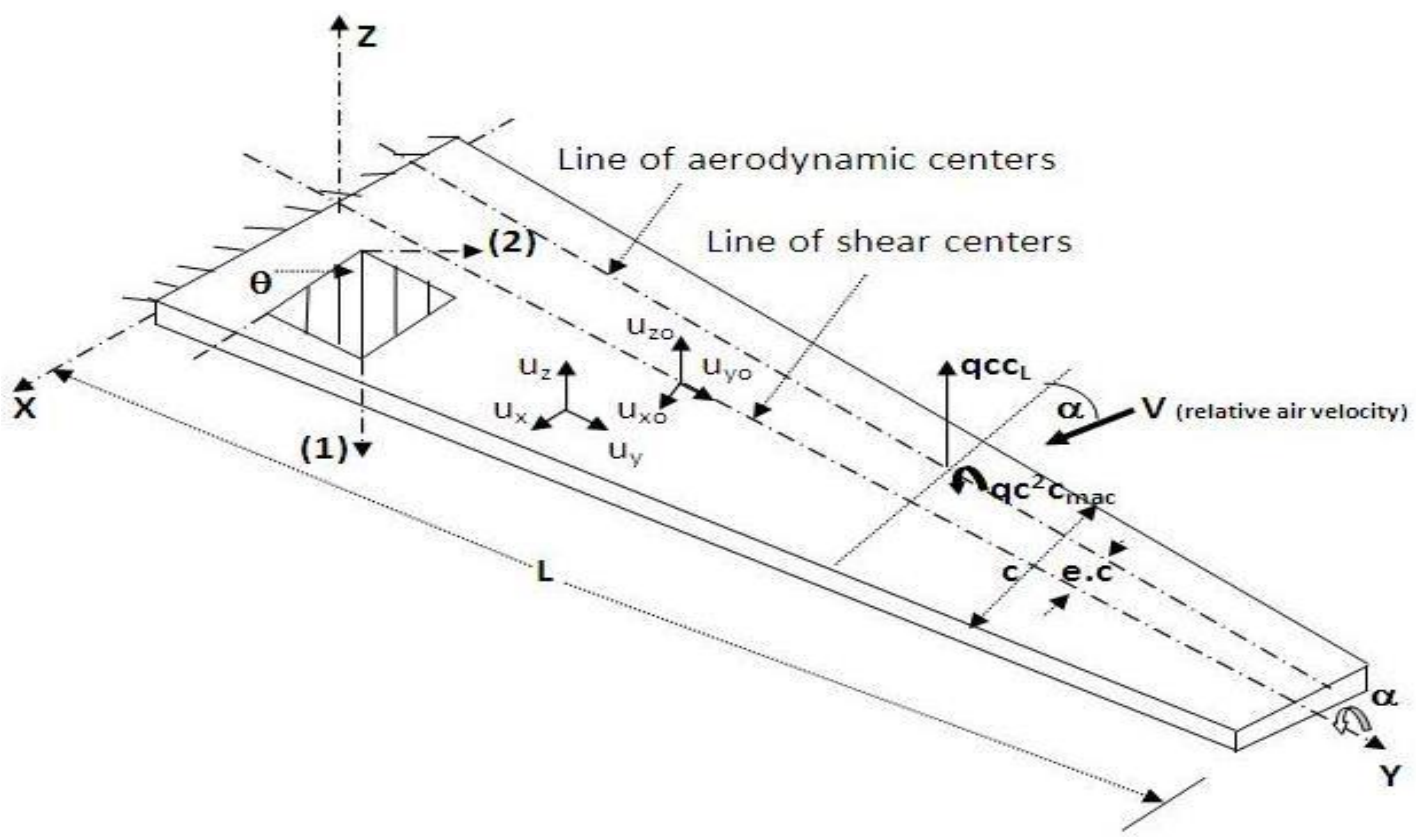

Fig. 1a. Plate-beam wing model and definition of reference axes.
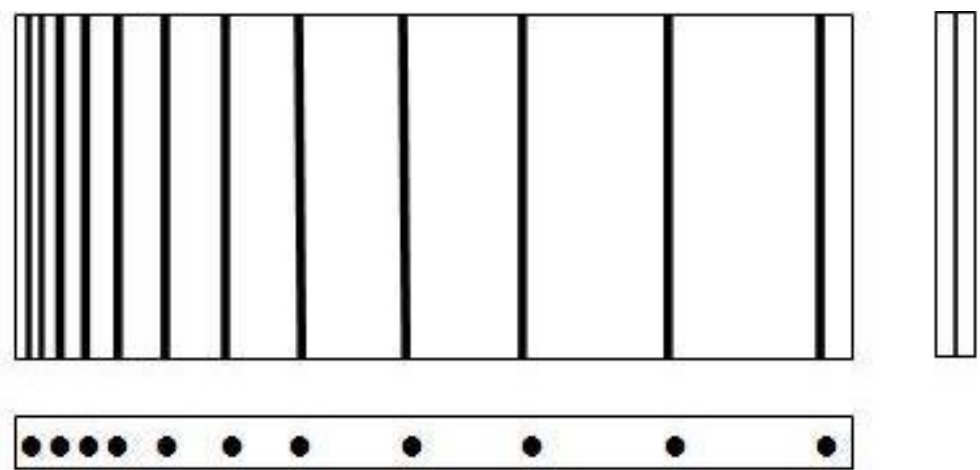

Fig. 1b. Spanwise grading of fibers in a fibrous composite plate. 
The strain energy stored in the wing structure is given by:

$$
U(y)=\frac{1}{2} E I \grave{u}_{z o}^{2}-K \grave{u}_{z o} \grave{\alpha}+\frac{1}{2} G J \grave{\alpha}^{2}
$$

The equivalent stiffness terms appearing in Eq.(1) are defined in the following:

Bending stiffness: $\quad: \quad E I=C\left(D_{22}-\frac{B_{22}^{2}}{A_{22}}\right)$

Torsional stiffness: $\quad: \quad G J=4 C\left(D_{66}-\frac{B_{26}^{2}}{A_{22}}\right)$

Bending-torsion coupling: $\quad K=2 C\left(D_{26}-\frac{B_{22} B_{26}}{A_{22}}\right)$

where:

Extensional stiffness:

$$
A m n=\sum_{i=1}^{N_{L}} \bar{Q}_{m n}^{(i)} h_{i}
$$

Bending-extensional coupling stiffness: $\quad B_{m n}=\sum_{i=1}^{N_{L}} \bar{Q}_{m n}^{(i)} \beta_{i}$

Bending stiffness:

$$
D_{m n}=\sum_{i=1}^{N_{L}} \bar{Q}_{m n}^{(i)} \delta_{i}
$$

where $(m, n=1,2,6)$ and $h_{i}$ is the thickness of the $i$-th lamina.

The distribution of the external work, $W_{\text {ext }}$, done by the aerodynamic loads along the wing per unit span is $[1,10]$ :

$$
W_{e x t}(y)=1 / 2\left(q c a \alpha u_{z o}+q c^{2} e a \alpha^{2}\right)
$$

where $q\left(=1 / 2 \rho_{\text {air }} V^{2}\right)$ is the dynamic pressure, $\rho_{\text {air }}$ is the air density at the specified flight altitude and $V$ the relative air speed. The term " $a$ " is the two-dimensional lift curve slope of the wing sections, which is empirically corrected to reflect the effect of finite span. Since the present study considers only low subsonic flight regime, no modification of the two-dimensional lift curve slope to account for compressibility is made. The symbol " $e$ " denotes the dimensionless distance, as a percentage of the wing chord, between the aerodynamic center $(A . C)$ and shear center (S.C), measured positive aft from the (A.C).

The finite element method (FEM) is an approximate technique that provides solutions for differential and integral equations, which may otherwise be impossible to solve in a closed-form [12]. The FEM is particularly useful in problems with complex geometry, loading and stiffness variations, such as variable-stiffness composite wings. For structures, the general approach is to divide a single complex geometry into a defined group of smaller, simpler shapes known as "finite elements". The continuous displacements are approximated by a discrete model composed of a set of piecewise functions defined using the values of the displacements at a finite number of points (called nodal points). The most popular form of the element function is the polynomial with an order depending on the number of degrees of 
freedom at each nodal point. In the present study the idealized built-up wing structure is divided into one-dimensional beam elements positioned along the elastic $y$-axis. Each element with length $L_{e}$ has two nodes, denoted with " 1 " and "2". Each node has three degrees of freedom: transverse translation $u_{z o}$, bending rotation $\varphi$ $\left(=d u_{z o} / d y\right)$ and torsional twist $\alpha$, as shown in figure (2).

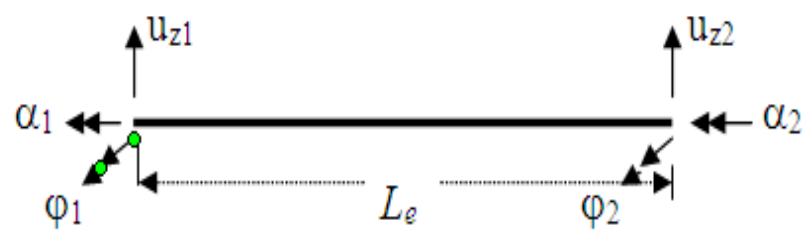

Fig. 2. Finite element degrees of freedom: equivalent beam model.

The continuous displacement functions $u_{z o}$ and $\alpha$ can be expressed, in terms of the nodal degrees of freedom, as follows [12]:

$$
\begin{gathered}
u_{z o}(\zeta)=\left(1-3 \zeta^{2}+2 \zeta^{3}\right) u_{z 1}+L_{e}\left(\zeta-2 \zeta^{2}+\zeta^{3}\right) \varphi_{1}+\left(3 \zeta^{2}-2 \zeta^{3}\right) u_{z 2}+L_{e}\left(\zeta^{3}-\zeta^{2}\right) \varphi_{2} \\
\alpha(\zeta)=(1-\zeta) \alpha_{1}+(\zeta) \alpha_{2} \quad, 0 \leq \zeta\left(=y / L_{e}\right) \leq 1
\end{gathered}
$$

Divergence is the case of neutral stability defined by the condition that the change in strain energy is equal to the work done by the aerodynamic loads during an arbitrary finite increase in the elastic deformations [13]. The total strain energy and external work done by air loads can be expressed in terms of the nodal degrees of freedom by substituting from equation (4) into equations (1) and (3), and integrating over the element length, $L_{e}$. To obtain the equations of equilibrium for the finite elements composing the wing structure, we use the principle of stationary total potential, $\pi$, i.e.

$$
\delta \pi=\delta \int_{0}^{1}\left(U-W_{e x t}\right) d \zeta=0
$$

where " $\delta$ " stands for an arbitrary variation in $\pi$ due to arbitrary variations in the nodal displacements satisfying the essential boundary conditions [12]. Using Eqs. (1), (3) and (4) in (5), integrating the displacement gradients by parts, and setting the coefficients of $\left(\delta u_{z}, \delta \varphi, \delta \alpha\right)_{j=1,2}$ to zero separately, the final normalized matrix form of the resulting equations which govern the static equilibrium of an element is cast in the following form:

$$
\left(\left[K^{e}\right]-\hat{q}\left[K_{A}^{e}\right]\right)\left\{U^{e}\right\}=\{0\}
$$

where $\left\{U^{e}\right\}$ is the nodal displacement vector $\left\{v_{z 1}, \varphi_{1}, \alpha_{1}, v_{z 2}, \varphi_{2}, \alpha_{2}\right\}^{T}$ and $v_{z j}\left(=u_{z j} / L\right)$ is a dimensionless bending displacement. $\left[K^{e}\right]$ is the element stiffness matrix normalized with respect to the torsional stiffness at the wing root (GJ) 0 of a baseline design. The latter is made of a cross-ply fibrous composite with equal volume fractions of the matrix and fiber materials (i.e. $V_{f 0}=50 \%$ ). The optimum wing designs 
studied herein will have the same length, chord distribution, cross section geometry, and type of fiber and matrix materials as those of the baseline design. The derived stiffness matrix in a normalized form is given be:

$$
\left[K^{e}\right]=\left[\begin{array}{cccccc}
12 K_{b} / \hat{L}_{e}^{2} & 6 K_{b} / \hat{L}_{e} & 0 & -12 K_{b} / \hat{L}_{e}^{2} & 6 K_{b} / \hat{L}_{e} & 0 \\
6 K_{b} / \hat{L}_{e} & 4 K_{b} & -K_{c} & -6 K_{b} / \hat{L}_{e} & 2 K_{b} & K_{c} \\
0 & -K_{c} & K_{t} & 0 & K_{c} & -K_{t} \\
-12 K_{b} / \hat{L}_{e}^{2} & -6 K_{b} / \hat{L}_{e} & 0 & 12 K_{b} / \hat{L}_{e}^{2} & -6 K_{b} / \widehat{L}_{e} & 0 \\
6 K_{b} / \hat{L}_{e} & 2 K_{b} & K_{c} & -6 K_{b} / \hat{L}_{e} & 4 K_{b} & -K_{c} \\
0 & K_{c} & -K_{t} & 0 & -K_{c} & K_{t}
\end{array}\right]
$$

where $K_{b}, K_{t}$ and $K_{c}$ are normalized stiffnesses defined as follows:

$$
\begin{aligned}
K_{b} & =\widehat{E I} / \widehat{L}_{e}, \widehat{E I}=E I / G J_{0} \\
K_{t} & =\widehat{G J} / \widehat{L}_{e}, \widehat{G J}=G J / G J_{0} \\
K_{c} & =\widehat{K} / \widehat{L}_{e}, \quad \widehat{K}=K / G J_{0}
\end{aligned}
$$

The dimensionless element length $\hat{L}_{e}$ is obtained by dividing its dimensional value $L_{e}$ by the total length of the wing, i.e. $\hat{L}_{e}=L_{e} / L$. The matrix $\left[K_{A}^{e}\right]$ is called the aerodynamic matrix depending upon the wing chord distribution, aspect ratio and the fractional location of the shear center with respect to the aerodynamic center. Its elements are defined by the following $(6 \times 6)$ square matrix:

$$
\left[K_{A}^{e}\right]=\left[\begin{array}{cccccc}
0 & 0 & \frac{7}{20} f \hat{C} \hat{L}_{e} & 0 & 0 & \frac{3}{20} f \hat{C} \hat{L}_{e} \\
0 & 0 & \frac{1}{20} f \hat{C} \hat{L}_{e}{ }^{2} & 0 & 0 & \frac{1}{30} f \hat{C} \hat{L}_{e}{ }^{2} \\
\frac{7}{20} f \hat{C} \hat{L}_{e} & \frac{1}{20} f \hat{C} \hat{L}_{e}{ }^{2} & \frac{2}{3} \hat{C}^{2} \hat{L}_{e} & \frac{3}{20} f \hat{C} \hat{L}_{e} & \frac{-1}{30} f \hat{C} \hat{L}_{e}{ }^{2} & \frac{1}{3} \hat{C}^{2} \hat{L} e \\
0 & 0 & \frac{3}{20} f \hat{C} \hat{L}_{e} & 0 & 0 & \frac{7}{20} f \hat{C} \hat{L}_{e} \\
0 & 0 & -\frac{1}{30} f \hat{C} \hat{L}_{e}{ }^{2} & 0 & 0 & -\frac{1}{20} f \hat{C} \hat{L}_{e}{ }^{2} \\
\frac{3}{20} f \hat{C} \hat{L}_{e} & \frac{1}{30} f \hat{C} \hat{L}_{e}{ }^{2} & \frac{1}{3} \hat{C}^{2} \hat{L} e & \frac{7}{20} f \hat{C} \hat{L}_{e} & \frac{-1}{20} f \hat{C} \hat{L}_{e}{ }^{2} & \frac{2}{3} \hat{C}^{2} \hat{L}_{e}
\end{array}\right]
$$

where $\hat{C}$ is a dimensionless chord length $\left(=C / C_{0}\right)$, and $f$ is a factor accounting for the effect of wing planform geometry:

$$
f=A R\left(1+\Delta_{c}\right) / 4 e
$$

$A R$ and $\Delta_{c}$ are the wing aspect and taper ratios, respectively, and e determines the location of the shear center with respect to the aerodynamic center. The quantity $\hat{q}$ in Eq.(6) is the dimensionless dynamic pressure defined by:

$$
\hat{q}=q \frac{e a C_{0}^{2} L^{2}}{2 G J_{0}}
$$

The entire or global stiffness and aerodynamic matrices can be obtained by summing up the individual matrices of each element given in Eqs. (7) and (9). Applying the boundary 
conditions at wing root $(y=0)$, namely; $u_{z 0}=\varphi=\alpha=0$, the associated eigenvalue problem of wing divergence is described by the following matrix equation:

$$
\frac{1}{\hat{q}}\{U\}=[K]^{-1}\left[K_{A}\right]\{U\}
$$

where $[K]$ and $\left[K_{A}\right]$ are the global stiffness and aerodynamic matrices, respectively. In order to calculate the critical flight speed at which wing divergence occurs, the largest eigenvalue $(1 / \hat{q})$ is taken leading to the smallest dynamic pressure at which static instability occurs. The normalized divergence speed can be calculated from:

$$
\widehat{V}_{D}=\sqrt{2 \hat{q}}
$$

where the normalized air speed is defined by the relation: $\widehat{V}=V C_{0} L \sqrt{\rho_{\text {air }} e a / 2 G J_{0}}$ Finally, it should be mentioned that for the case of cross-ply layup with $0^{\circ}$ and $90^{\circ}$ fiber orientation angles, the torsion and bending degrees of freedom become uncoupled, and only torsional divergence can be realized. In such a special case the element stiffness and aerodynamic matrices reduce to the following forms:

Stiffness matrix:

$$
\left[K^{e}\right]=\left[\begin{array}{rr}
K_{t} & -K_{t} \\
-K_{t} & K_{t}
\end{array}\right]
$$

Aerodynamic matrix:

$$
\left[K_{A}^{e}\right]=\left[\begin{array}{cc}
\frac{2}{3} \hat{C}^{2} \hat{L}_{e} & \frac{1}{3} \hat{C}^{2} \hat{L} e \\
\frac{1}{3} \hat{C}^{2} \hat{L} e & \frac{2}{3} \hat{C}^{2} \hat{L}_{e}
\end{array}\right]
$$

\section{CONTINUOUS MODELS}

For continuous grading models described by power-law expressions (refer to Table .1), only one wing panel constructed from just one composite layer with changing fiber volume fraction is considered to perform design optimization. In such a cases design variables will include the taper ratio of fiber volume fraction, $\Delta_{t}$, the power exponent, $p$, and ply angle, $\theta$. The equality mass constraint can be used to eliminate one of the design variables, which simplify the mathematical model and, consequently, reduce the needed computational time. Moreover, for cases with thickness grading, the wing cross section is subdivided into thinner strips, each of which has different properties determined according to the grading pattern. The classical lamination theory can still be applied, where the thin strips may be imagined as if they were perfectly-bonded laminae composing the wing section. Table (1) describes the various forms of the design variable vector $\underline{X}$ for different grading patterns. Therefore, for continuous grading models, the associated optimization problem is cast in the following form:

Find the design variables vector $\underline{X}=\left(\Delta_{f}, p, \theta\right)$ which minimizes the objective function:

$$
F(\underline{X})=-\widehat{V}_{D}
$$

subject to the constraints: 


$$
\begin{gathered}
\widehat{M}_{s}=1 \\
\Delta_{f}^{(L)} \leq \Delta_{f} \leq \Delta_{f}^{(U)} \\
\theta^{(L)} \leq \theta \leq \theta^{(U)} \\
P \geq 0
\end{gathered}
$$

while the normalized structural mass $\left(\widehat{M}_{S}\right)$ can be obtained by dividing the total mass by that of the baseline design, to get:

$$
\widehat{M}_{s}=\frac{3}{\left(1+\Delta_{c}+\Delta_{c}^{2}\right)} \sum_{j=1}^{N_{E}} \hat{C}_{j} \hat{L}_{j} \sum_{i=1}^{N_{L, j}} \hat{\rho}_{i j} \hat{h}_{i j}
$$

where

$$
\widehat{M}_{s}=M_{s} / M_{0} \text { and } \hat{\rho}_{i j}=\rho_{i j} / \rho_{0} .
$$

Table 1. Design variables for different grading patterns.

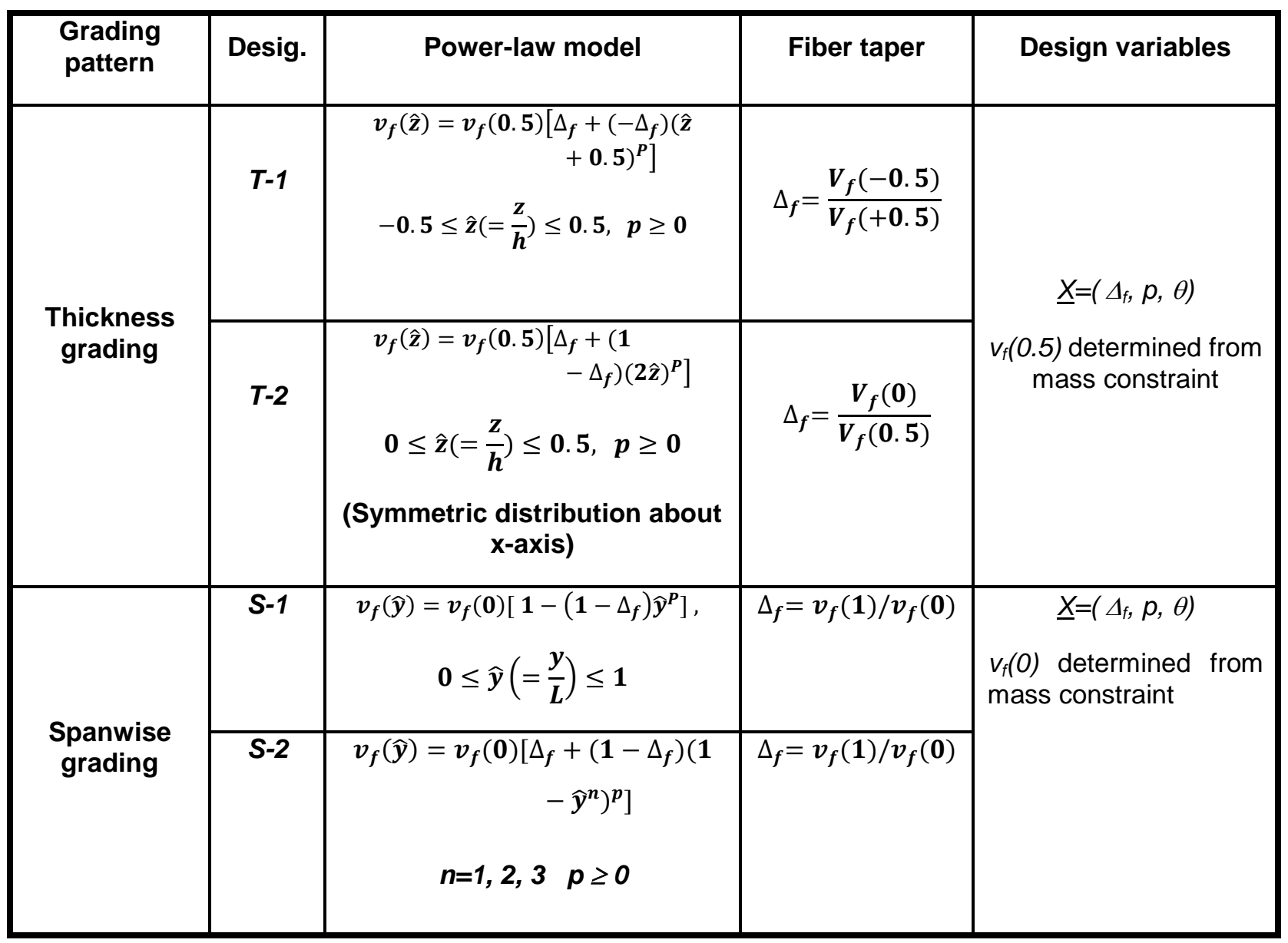




\section{GENERAL DESCRIPTION AND TECHNICAL DATA OF THE WING BASELINE DESIGN}

In this study the wing baseline design has a trapezoidal shape with zero sweep angle and rigid twist. The main construction is made of cross-ply $\left[0^{\circ}, 90^{\circ}\right]_{s}$ layup with equal fiber and matrix volume fractions, i.e. $V_{f_{o}}=V_{m o}=50 \%$. Other pertinent data are given in the following:

Wing span $(2 L=9.60 \mathrm{~m})$, Wing gross area $\left(S_{w}=11.80 \mathrm{~m}^{2}\right)$, Aspect ratio $(A R=$ 7.81), Structural mass ( $\left.M_{o}=500 \mathrm{~kg}\right), 2 D$ lift-curve slope $\left(a_{o}=2 \pi\right)$, Flight altitude $3 \mathrm{~km}$ $\left(\rho_{\text {air }}=0.9093 \mathrm{~kg} / \mathrm{m}^{3}\right)$, Maximum flight speed $\left(V_{\max }=108 \mathrm{~m} / \mathrm{s}\right)$.

The type of material of construction is selected to be carbon-AS4/epoxy-3501-6 composite, which has favorable characteristics and is highly desirable in both civilian and military aircraft structures. Its properties are given in Table 2. Moreover, for a flat thin plate wing, the offset between the aerodynamic and elastic axes " $e$ " is taken to be $25 \%$ of the chord length. The corrected $3 D$ curve-lift slope was calculated to be 5.0 .

For a given value of the taper ratio " $\Delta_{c}$ ", the chord at wing root " $C_{0}$ " can be determined from:

$$
C_{0}=\frac{S_{w}}{L\left(1+\Delta_{c}\right)}
$$

The height, or total thickness, of the wing cross section at root can be calculated from the expression:

$$
H_{0}=\frac{3 M_{o}}{\rho_{o} C_{o} L\left(1+\Delta_{c}+\Delta_{c}^{2}\right)}
$$

where $\rho_{0}=0.5\left(\rho_{f}+\rho_{m}\right)=1540 \mathrm{~kg} / \mathrm{m}^{3}$.

Table 2. Material properties of carbon-AS4/ epoxy-3501-6 composite[11].

\begin{tabular}{|c|c|c|}
\hline Property & Carbon fiber & $\begin{array}{c}\text { Epoxy } \\
\text { matrix }\end{array}$ \\
\hline Mass density $\left(\mathrm{g} / \mathrm{cm}^{3}\right)$ & $\rho_{f}=1.81$ & $\rho_{m}=1.27$ \\
\hline Young's moduli $(\mathrm{Gpa})$ & $E_{1 f}=235, E_{2 f}=15$ & $E_{m}=4.3$ \\
\hline Shear moduli $(\mathrm{Gpa})$ & $\mathrm{G}_{12 f}=27, \mathrm{G}_{23 f}=7$ & $\mathrm{G}_{m}=1.60$ \\
\hline Poisson's ratio & $v_{12 f}=0.2$ & $v_{m}=0.35$ \\
\hline Ultimate tensile strength $(\mathrm{MPa})$ & 3700 & 69 \\
\hline Ultimate compressive strength $(\mathrm{MPa})$ & - & 200 \\
\hline Ultimate shear strength $(\mathrm{MPa})$ & - & 100 \\
\hline
\end{tabular}


Substituting for the prescribed values of the quantities $L, S_{w}, M_{o}$ the cross sectional dimensions and the torsional rigidity at root of the wing baseline design for different taper ratios are given in Table (3). Actually these values belong to a family of wing baseline designs with different chord taper but having the same aspect ratio, wing area and total structural mass. The average value of the airfoil thickness-to-chord ratio at wing root is about $4.25 \%$.

Table 3. Root torsional rigidity and divergence speed for different wing taper.

\begin{tabular}{|c|c|c|c|c|c|}
\hline $\boldsymbol{\Delta}_{c}$ & $\boldsymbol{C}_{o}(\boldsymbol{m})$ & $\boldsymbol{H}_{o} \times \mathbf{1 0}^{-2}(\boldsymbol{m})$ & $G_{J_{o}} \times \mathbf{1 0}^{4} \mathbf{N} \cdot \boldsymbol{m}^{2}$ & $\widehat{V}_{D o}$ & $V_{D o} \mathbf{m} / \mathbf{s}$ \\
\hline 0.25 & 1.97 & 7.85 & 132.01 & 1.68 & 270.79 \\
\hline 0.5 & 1.64 & 7.07 & 80.29 & 1.65 & 249.14 \\
\hline 0.75 & 1.405 & 6.25 & 47.52 & 1.61 & 218.3 \\
\hline 1.0 & 1.23 & 5.5 & 28.35 & 1.57 & 187.82 \\
\hline
\end{tabular}

The last two columns in Table (3) give the normalized divergence speed and the actual dimensional value as calculated by using equation (Eq. (13)).

Figure (3) shows variation of the normalized and actual dimensional divergence speed with chord taper for such a family of wing baseline designs. It is seen that the dimensionless divergence speed is slightly decreasing with chord taper. This is merely due to the scaling effect considered in the mathematical model formulation, which makes most of the problem variables and parameters have close order of magnitudes. The actual dimensional speed can be obtained by multiplying its dimensionless value by the factor: $\frac{1}{C_{o} L} \sqrt{\frac{2 G J_{o}}{\rho_{\text {air } e a}}} \mathrm{~m} / \mathrm{s}$ (refer to Eq.13), which can be determined from the known baseline design parameters. It is observed that the actual divergence speed is decreasing sharply with increasing chord taper, which is a naturally expected behavior.

\section{RESULT OF CONTINUOUS SPANWISE GRADING, (S-1) - GRADING MODEL [9]}

The first case study to be implemented is designated by the $(S-1)$-grading model, which considers variation of the fiber volume fraction in the spanwise direction according to a power-law expression. The wing is constructed from just one panel with cross-ply $\left[0^{\circ}, 90^{\circ}\right]_{s}$ layup. The structure was divided into 15 finite elements of equal length. Figure (4) shows variation of the resulting optimal solutions $\underline{X}=\left(\Delta_{f}, p\right)_{\text {opt }}$ and $\hat{V}_{D, \max }$ with the wing chord tapering ratio, $\Delta_{c}$. It is seen that the maximum normalized divergence speed, $\widehat{V}_{D, \max }$ changes smoothly with $\Delta_{c}$ around an average value of 1.72. Wings having small chord taper possess higher aeroelastic stability, which is an expected natural behavior. The optimal power-exponent " $p$ " curve is seen to be split into two curves asymptotic to the vertical line $\Delta_{c}=\Delta_{c s} \cong 0.56$. In the range $0.25 \leq \Delta_{c} \leq 0.4$, ' $p$ ' slightly increases around an average value of 0.5 , while the fiber volume fraction ratio $\Delta_{f}=v_{f t} / v_{f r}$ remains constant at its maximum value of 3.0. This 


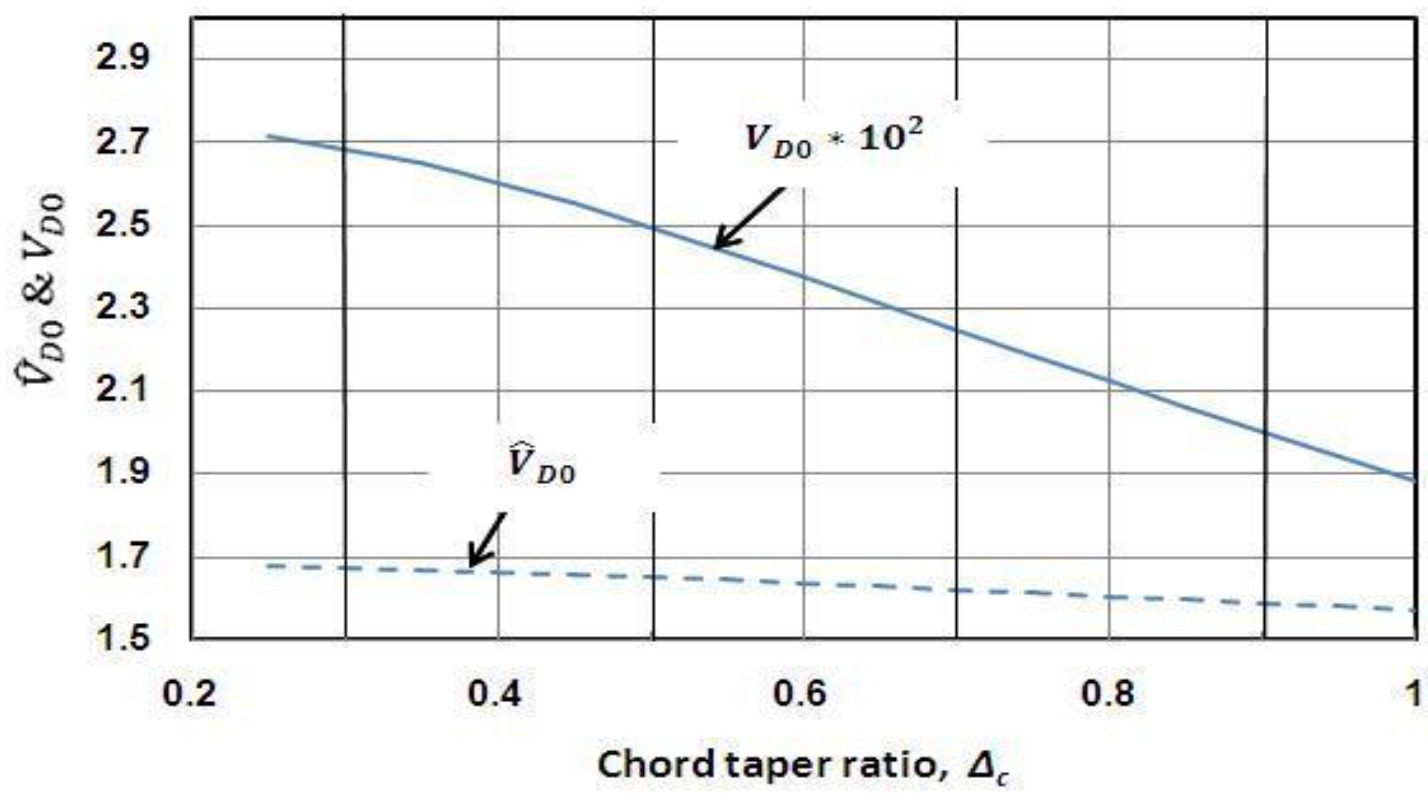

Fig. 3. Variation of dimensionless and actual dimensional divergence speed of the wing baseline design with chord taper (Same $A R, S_{w}$ and $M_{o}$ ).

means that the fiber volume fraction reaches its lower limiting value at wing root and its upper value at wing tip inside this range. For $0.4 \leq \Delta_{c}<\Delta_{c s}$, both $\left(\Delta_{f}, p\right)_{\text {opt }}$ decrease towards the separation point $\Delta_{c s} \cong 0.56$, where a jump in the power exponent curve can be noticed near the vertical line $\Delta_{c s} \cong 0.56$. For the range $\Delta_{c}>$ $\Delta_{c s}, p$ decreases rapidly reaching a value of 1 corresponding to a rectangular wing planform. At the same time, the optimal volume fraction ratio $\Delta_{f}$ changes slightly around an average value of 0.35 . Numerical results of the final optimal designs are given in Table 4 for some selected values of $\Delta_{c}$.

It should be reminded that such a behavior depends entirely upon the pre-assigned mathematical form of the power-law expression, which represents an additional constraint on the proposed optimization model. The problem of determining the global optimal distribution of the volume fraction may be treated using optimal control theories [15]. Such a sophisticated mathematical problem is out of the scope of the present work.

\section{RESULT OF (S-2)-GRADING MODEL [16]}

The second case study is designated by the (S-2)-grading model, which considers variation of the fiber volume fraction along the wing span according to Table 1. Figure (5) depicts the attained optimal solutions with varying chord taper ratio for the linear case, where the coordinate exponent $n=1$. The maximized objective function, $\hat{V}_{D, \max }$, retains similar variation with $\Delta_{c}$ as that obtained by using the ' $S-1$ 'grading model (see Fig. 4). However, the two branches of the optimal power exponent, $p$ curve, are seen to be reversed, but with nearly similar behavior to that obtained in 


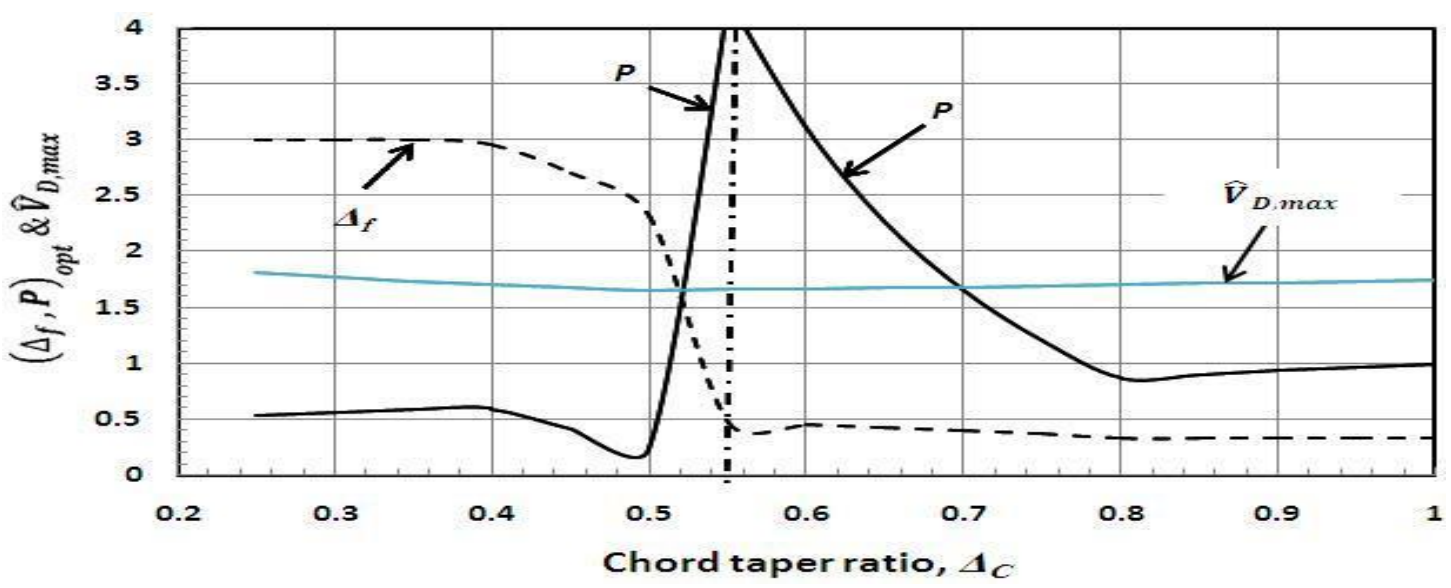

Fig. 4. Variation of optimum design solution $\left(\Delta_{f}, p\right)_{\text {opt }}$ and $\widehat{V}_{D, \max }$, with chord taper ratio, $\Delta_{c}$ : Case of spanwise ' $S-1$ ' material grading model.

Table 4. Optimal wing designs using spanwise ' $S-1$ ' grading model.

\begin{tabular}{|c|c|c|c|c|c|}
\hline$\Delta_{C}$ & $\left(\Delta_{f}, \boldsymbol{p}\right)_{\text {opt. }}$ & $\left(\boldsymbol{v}_{f r}, \boldsymbol{v}_{\boldsymbol{f f}}\right)_{\text {opt }}$ & $\widehat{\boldsymbol{V}}_{\boldsymbol{D}, \max }$ & $\boldsymbol{V}_{\boldsymbol{D}, \max }(\mathbf{m} / \mathbf{s})$ & Gain\% \\
\hline 0.25 & $(3.0,0.535)$ & $(0.25,0.75)$ & 1.811 & 292.7 & 7.8 \\
\hline 0.5 & $(2.32,0.236)$ & $(0.25,0.58)$ & 1.66 & 250.65 & 0.5 \\
\hline 0.55 & $(0.47,4.25)$ & $(0.53,0.25)$ & 1.67 & 247.5 & 1.6 \\
\hline 0.75 & $(0.373,1.205)$ & $(0.671,0.25)$ & 1.691 & 229.1 & 4.8 \\
\hline 1.0 & $(0.333,1.0)$ & $(0.75,0.25)$ & 1.748 & 209.6 & 11.4 \\
\hline
\end{tabular}

the 'S-1'model. The separation point, $\Delta_{c s}$ is seen to be shifted slightly to the left, where the two branches become asymptotic to the vertical line $\Delta_{c s} \cong 0.44$. On the other hand, the optimal fiber ratio $\Delta_{f}$ retains the same behavior reaching its minimum value of 0.33 when the wing planform becomes rectangular, i.e. $\Delta_{c}$ approaches unity. The case of $n=2$ is shown in Fig. 6 . It is seen that the $\Delta_{f}$-curve retains a similar shape to that obtained before in the other cases. The optimization favors more fibers near the wing root for chord taper ratios greater than 0.5. A more expanded stability boundary than that obtained in the case of $n=1$ can also be observed, where higher values of the maximized objective function $\widehat{V}_{D, \max }$ are achieved. The optimal power exponent curve is seen to be asymptotic to the vertical line $\Delta_{c s} \cong 0.425$. However, the obtained values of the power exponent ' $p$ ' are seen to be much higher than those obtained in the case of $n=1$.

Table (5) gives the attained optimal solutions using the ' $S-2$ grading model for different values of the coordinate exponent ' $n$ ' and chord taper ratio ' $\Delta_{c}$ '. As a general observation, the achieved optimization gain in the divergence speed increases with $n$ 


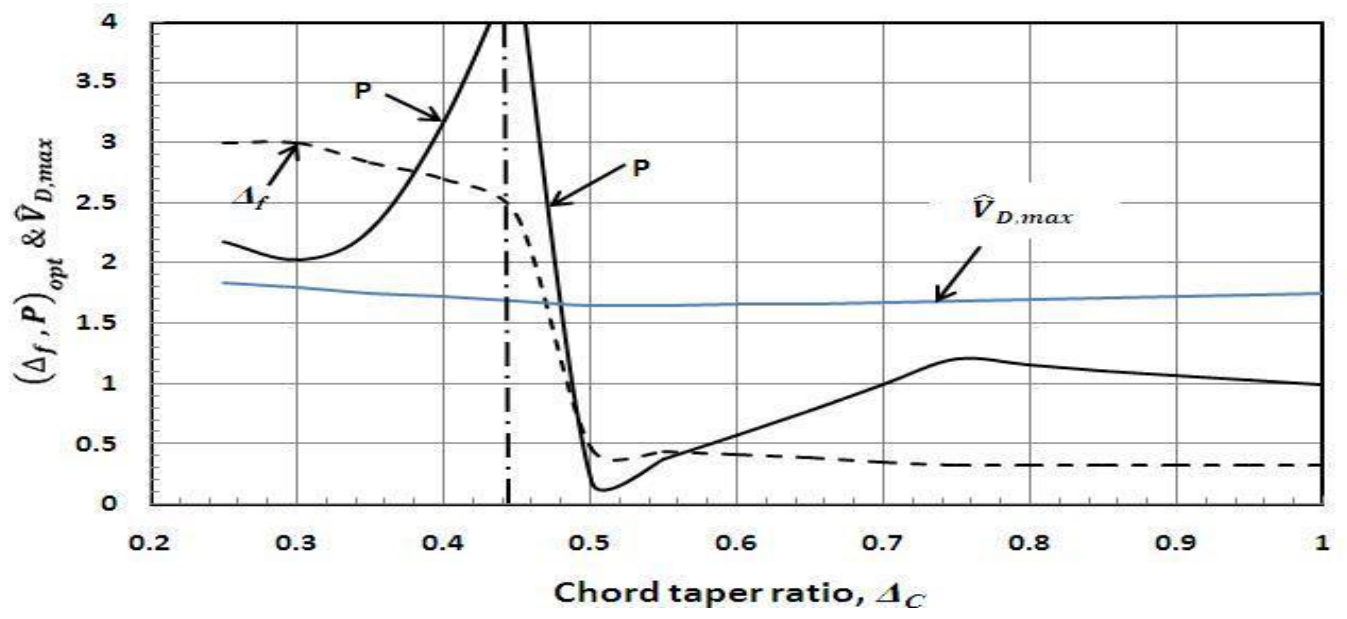

Fig. 5. Variation of $\left(\Delta_{f}, p\right)_{\text {opt }}$ and $\widehat{V}_{D, \max }$, with chord taper ratio, $\Delta_{c}$ Case of spanwise ' $S$-2' model $(n=1)$.

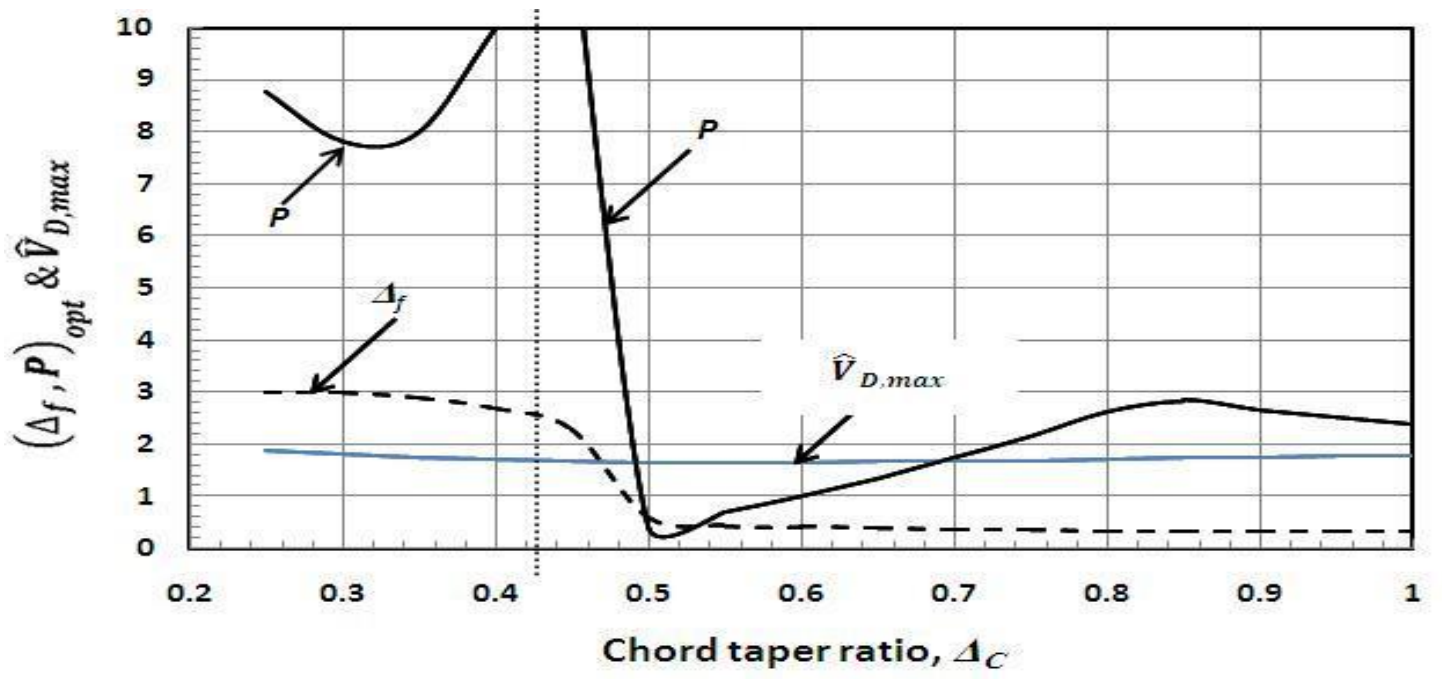

Fig. 6. Variation of $\left(\Delta_{f}, p\right)_{\text {opt }}$ and $\widehat{V}_{D, \max }$, with chord taper ratio, $\Delta_{c}$ Case of spanwise ' $S$-2' model $(n=2)$.

in most cases, reaching a value of $15.1 \%$ at $n=3$ for wings having a rectangular shape. It is also remarked that all spanwise grading models fail to give any significant improvement in the aeroelastic performance in the neighborhood of $\Delta_{c}=$ 0.5 , where the obtained optimization gain is almost less than $1 \%$.

Table (6) summarizes the actual values of the optimal fiber volume fractions at wing root and tip, as well as the maximum divergence speed $V_{D \text {,max }}$ in $\mathrm{m} / \mathrm{s}$. It is seen that all values of $V_{D, \max }$ are much higher than those of the corresponding baseline designs for different chord taper ratios. 
Table 5. Optimal solutions using spanwise ' $S-2$ ' grading model

\begin{tabular}{|c|c|c|c|}
\hline \multirow{2}{*}{$\Delta_{C}$} & \multicolumn{3}{|c|}{$\left(\Delta_{f}, p\right)_{\text {opt. }}, \hat{V}_{D, \max }, \quad$ Gain \% } \\
\cline { 2 - 4 } & $n=1$ & $n=2$ & $n=3$ \\
\hline 0.25 & $(3.0,2.18), 1.845,9.8 \%$ & $(3.0,8.78), 1.87,11.5 \%$ & $(3.0,1.0), 1.84,9.3 \%$ \\
\hline 0.50 & $(0.47,0.2), 1.657,0.3 \%$ & $(0.47,0.43), 1.660,0.5 \%$ & $(0.47,0.717), 1.665,0.8 \%$ \\
\hline 0.55 & $(0.44,0.38), 1.66,0.8 \%$ & $(0.45,0.70), 1.66,1.1 \%$ & $(0.45,1.1), 1.67,1.5 \%$ \\
\hline 0.75 & $(0.33,1.21), 1.69,4.9 \%$ & $(0.365,2.18), 1.7,5.5 \%$ & $(0.38,3.6), 1.71,6.0 \%$ \\
\hline 1.0 & $(0.33,1.0), 1.75,11.4 \%$ & $(0.33,2.38), 1.79,13.8 \%$ & $(0.33,5.02), 1.81,15.1 \%$ \\
\hline
\end{tabular}

Table 6. Optimal volume fractions at wing root and tip: ' $S-2$ ' grading model

\begin{tabular}{|c|c|c|c|}
\hline \multirow{2}{*}{$\Delta_{c}$} & \multicolumn{3}{|c|}{$\left(v_{f r}, v_{f t}\right)_{\text {opt. }}, V_{D, \max } \mathrm{m} / \mathrm{s}$} \\
\cline { 2 - 4 } & $n=1$ & $n=2$ & $n=3$ \\
\hline 0.25 & $(0.25,0.75), 298.2$ & $(0.25,0.75), 302.7$ & $(0.26,0.75), 296.9$ \\
\hline 0.50 & $(0.534,0.25), 250.4$ & $(0.536,0.25), 251.1$ & $(0.536,0.25), 251.7$ \\
\hline 0.55 & $(0.57,0.25), 245.6$ & $(0.56,0.25), 246.5$ & $(0.56,0.25), 247.3$ \\
\hline 0.75 & $(0.75,0.25), 229.3$ & $(0.69,0.25), 230.7$ & $(0.66,0.25), 231.6$ \\
\hline 1.0 & $(0.75,0.25), 209.6$ & $(0.75,0.25), 214.2$ & $(0.75,0.25), 216.6$ \\
\hline
\end{tabular}

\section{Result of Continuous thickness grading}

Figure (7) shows variation of the optimal design variables $\left(\Delta_{f}, p\right)_{\text {opt, }}$ and maximum normalized divergence speed $\widehat{V}_{D, \max }$ with the chord taper ratio $\Delta_{c}$ for the case of thickness, ' $T$-1' grading model [17]. The wing is constructed from just one panel $\left(N_{p}=1\right)$ divided into 15 finite elements $\left(N_{E}=15\right)$ with equal lengths. Cross-ply $\left[0^{\circ}, 90^{\circ}\right]_{s}$ layup was considered, where each lamina at wing root is imagined to be subdivided into 5 thin perfectly-bonded layers of equal thicknesses, i.e. $\left(N_{L r}=20\right)$.

It is seen that better wing designs have an increasing convex variation of the power exponent ' $p$ ' with wing taper ' $\Delta_{c}$ '. Wings with sharp taper possess higher values of $\widehat{V}_{D, \max }$, which indicates more expanded aeroelatic stability boundary. Regarding variation of the volume fraction ratio ' $\Delta_{f}$ ', the optimization favors more fibers at the upper surface of the wing. The lower surface has less fiber volume fraction by about $45 \%$. 


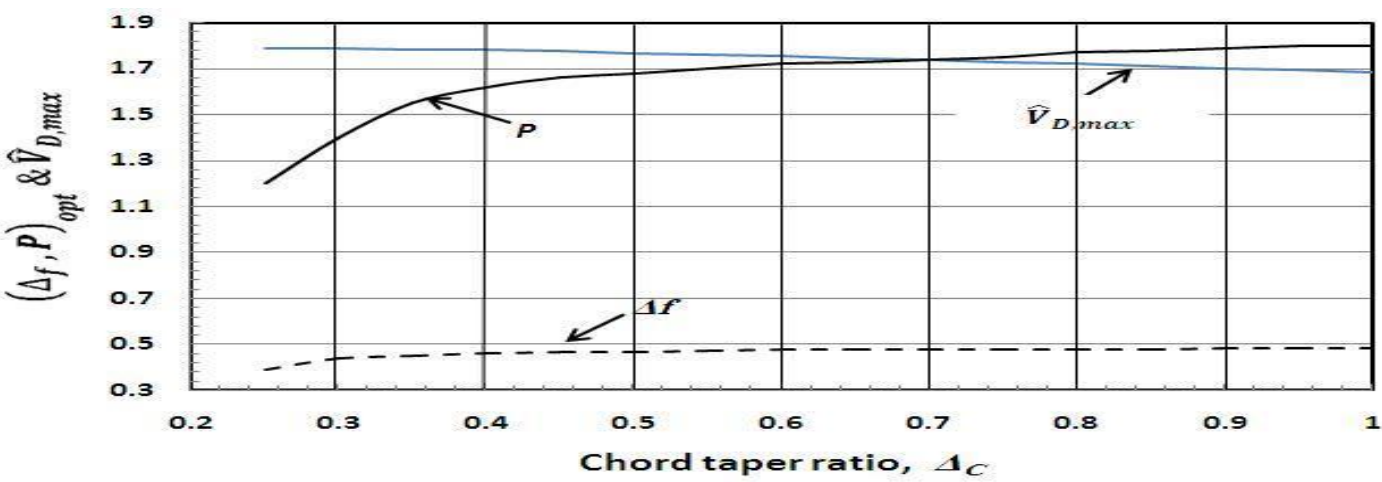

Fig.7. Variation of $\left(\Delta_{f}, p\right)_{\text {opt }}$ and $\widehat{V}_{D, \max }$, with chord taper ratio, $\Delta_{C}$ Case of thickness ' $T$-1' grading model.

Results for the case of symmetrical thickness grading ' $T$-2' [18] are shown in Figure (8). Better wing designs have an increasing concave variation of the power exponent ' $p$ ' with wing taper ' $\Delta_{c}$ '. The optimal fiber volume fraction ratio almost remains constant at an average value of 0.33 . This means that the volume fraction reaches its lower limiting value (25\%) at the middle surface of the wing and its upper value (75\%) at the upper and lower surfaces.

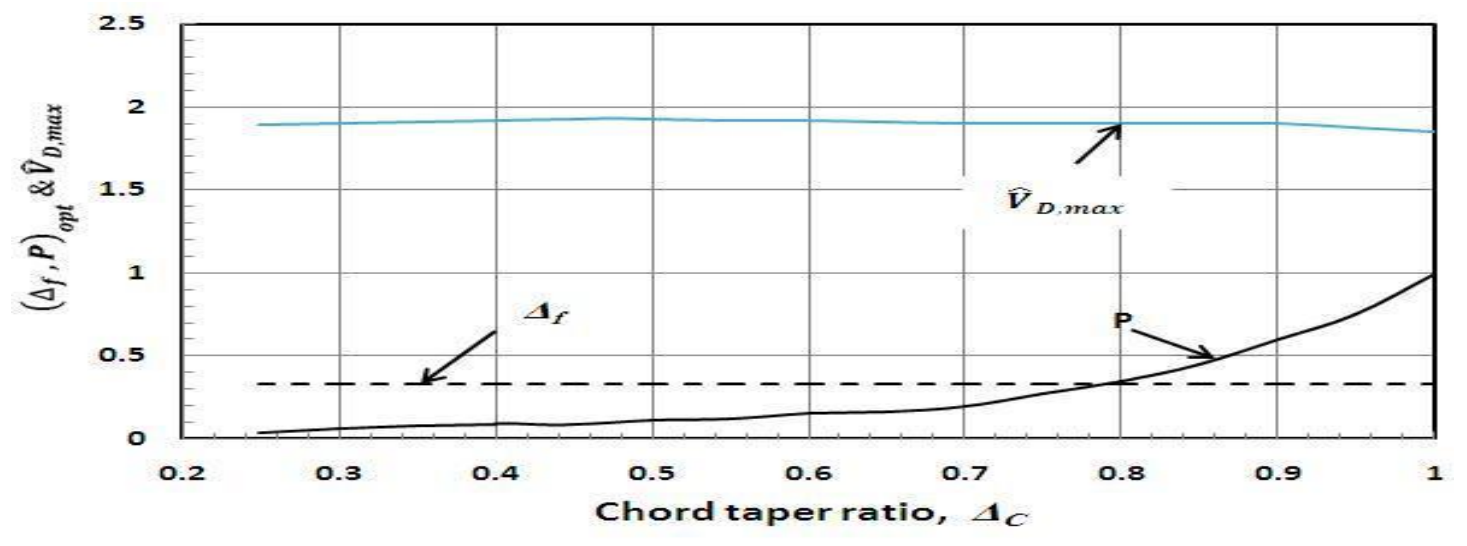

Fig.8. Variation of $\left(\Delta_{f}, p\right)_{\text {opt }}$ and $\widehat{V}_{D, \max }$, with chord taper ratio, $\Delta_{C}$ Case of thickness ' $T$-2' grading model.

Table (7) summarizes the attained optimal solutions for both thickness ' $T-1$ ' and ' $T-2$ ' grading models. It is obvious that symmetric material grading, as described by the ' $T$ 2 ' model, results in better wing designs having higher values of the maximum divergence speed, $\widehat{V}_{D, \max }$. The optimal power exponent for the ' $T$-1' model always exceeds 1.0, which means that the volume fraction has convex variation in the airfoil thickness direction. In contrast to this, the power exponent is less than or equal to 1.0 for the symmetric thickness grading model, indicating concave variation of the volume fraction in the airfoil thickness direction. 
Table 7. Optimal solutions using thickness grading.

\begin{tabular}{|l|c|c|}
\hline \multirow{2}{*}{$\Delta_{c}$} & \multicolumn{2}{|c|}{$\left(\Delta_{f}, p\right)_{o p t}, \widehat{V}_{D, \text { max }}$, Gain\% } \\
\cline { 2 - 3 } & $(T-1)$ grading model & $(T-2)$ grading model \\
\hline 0.25 & $(0.392,1.20), 1.787,6.4 \%$ & $(0.333,0.04), 1.87,11.3 \%$ \\
0.5 & $(0.473,1.70), 1.761,7.1 \%$ & $(0.333,0.12), 1.97,19.3 \%$ \\
0.75 & $(0.478,1.75), 1.729,7.2 \%$ & $(0.333,0.28), 1.86,15.3 \%$ \\
1.0 & $(0.483,1.80), 1.685,7.2 \%$ & $(0.333,1.0), 1.85,17.7 \%$ \\
\hline
\end{tabular}

\section{CONCLUSIONS}

\section{The following conclusions are obtained:}

1. For the continuous spanwise grading models ' $S-1$ ' and ' $S-2$ ', it was found that the optimal power exponent curve consists of two branches asymptotic to a vertical line located at a specific value of the wing chord taper ratio (denoted by $\Delta_{c s}$ ) ranging between 0.42 and 0.56 . The optimization favors more dense fibers near the wing root for chord taper ratios greater than $\Delta_{c s}$.

2. For the ' $S-2$ ' model, more expanded stability boundaries have been achieved for higher values of the coordinate exponent ' $n$ ', where the normalized divergence speed reaches its uppermost levels. However, in the neighborhood of $\Delta_{c s}$, both power-law grading models fail to give any significant improvement in the aeroelastic performance, where the attained optimization gain is less than $1 \%$.

3. Concerning grading in the airfoil thickness direction described by the ' $T$ 1 'model, it was found that better wing designs have an increasing convex variation of the power exponent ' $p$ ' with wing taper.The attained numerical values always exceed 1.0, which means that the volume fraction has also convex variation in the airfoil thickness direction. Wings with sharp taper possess higher divergence speeds, which indicates more broadened aeroelatic stability boundaries. The optimization favors more dense fibers at the wing upper surface than at the lower one.

4. Symmetric material grading described by the ' $T-2$ 'model results in the highest stability level. It was shown that better wing designs have increasing concave variation of the optimal power exponent with wing taper. The attained numerical values were found to be less than or equal to 1.0, indicating concave variation of the volume fraction in the airfoil thickness direction. Results also show that optimization favors more fibers at the upper and lower surfaces of the wing with the volume fraction reaching its upper limit value of $75 \%$. At the middle surface, however, the optimal fiber volume fraction approaches its lower limit value of $25 \%$. 


\section{The following Recommendations are given in the following:}

1. The attained optimal solutions using continuous material grading depend entirely upon the pre-assigned mathematical form of the power-law expression, which represents an additional constraint on the proposed optimization model. The problem of determining the actual optimal distribution of the volume fraction may be treated using advanced optimal control theories.

2. Future study should apply multi-criteria optimization by maximizing both divergence and flutter speed under mass constraint.

3. The optimization analysis can be extended to cover other cross-sectional types of the wing, such as a rectangular, thin-walled box sections.

4. This paper deals with aeroelastic stability only. Response problem optimization will be considered in a future study.

\section{REFERENCES}

[1] Hodges D.H., Pierce G.A. "Introduction to structural dynamics and aeroelasticity," Cambridge University Press, $1^{\text {st }}$ Edition, 2002.

[2] Martins J.R., Alonso J., and Reuther J., "High-fidelity aero-structural design optimization of a supersonic business jet," Journal of Aircraft, Vol. 41, No.3, pp. 523-530, 2004.

[3] Bulter R., Lillico M., Banerjee J.R. and Gum S., "Optimum design of high aspect ratio wing subject to aeroelastic constraints," AIAA/ASME/ASCE/AHS, Structure, Structural Dynamics and Materials Conference, New York, pp. 558566, 1995.

[4] Peter J.R., Dimitri N.M. and Daniel P.S., "Combined aerodynamic and structural optimization of a high-speed civil transport wing," AIAA paper-951222, 1995.

[5] Layton J.B., "Aeroelastic and aeroservoelastic tailoring with geometry variables for minimizing the gust response of a cantilevered finite span wing." AIAA/ASME/ASCE/AHS, Structure, Structural Dynamics and Materials Conference, New York, pp. 1086-1103, 1996.

[6] Suresh S. and Mortensen A., "Fundamentals of functionally graded materials" Cambridge, MA, USA, and Lausanne, Switzer Land, February 1998.

[7] Cho, J. R., and Oden, J. T., "Functionally graded material: A parametric study on thermal-stress characteristics using the Crank-Nicolson-Galerkin scheme," Comput. Methods Appl. Mech. Eng., 188, pp. 17-38, 2000.

[8] Cho, J. R., and Shin, S. W., "Material composition optimization for heatresisting, FGM by artificial neural network," Composites, Part A, 35, pp. 585594, 2004.

[9] Librescu L \& Maalawi K., "Material grading for improved aeroelastic stability of composite wings", Journal of Mechanics of Materials and Structures, Vol. 2, No.7, pp. 1381-1394, 2007.

[10] Librescu L. and Maalawi K.Y., "Aeroelastic design optimization of thin-walled subsonic wings against divergence," Thin-Walled Structures, Vol.47, pp.8997, 2009.

[11] Venkataraman P., "Applied optimization with MatLab programming", John Wiley \& Sons, Inc., New York, 2009. 
[12] Bathe KJ. Finite element procedures in engineering analysis. Prentice Hall, NJ, 1982.

[13] R. Bisplinghoff R.L., Ashley H. and Halfman R.L., "Aeroelasticity," Addison Wesley, 1955.

[14] Daniel, I.M. and Ishai, O., "Engineering mechanics of composite materials" $2^{\text {nd }}$ ed, Oxford Univ. Press, New York, 2006.

[15] Rao S., "Engineering optimization: theory and practice," $4^{\text {th }}$ edition, John Wiley \& Sons, ISBN: 978-0470183526, New York, 2009.

[16] Shin-Yao K., "Flutter of rectangular composite plates with variable fiber spacing," Composite Structures, Vol. 93, pp. 2533-2540, 2011.

[17] Lipton, R., "Design of functionally graded composite structures in the presence of stress constraints," Int. J. Solids Structures, Vol.39, pp. 25752586, 2002.

[18] Bedjilili Y., Tounsi A., Berrabah H.M. and Mechab I., "Natural frequencies of composite beams with a variable fiber volume fraction including rotary inertia and shear deformation," Applied mathematics and Mechanics, Vol. 30, No. 6, pp. 717-726, 2009. 